\title{
Nanoparticles: tech trends in healthcare
}

\author{
Anu Chandran', Varun Raghavan ${ }^{2}$, Bhaskaran Chalil ${ }^{3}$, Kamalasanan ${ }^{3}$, C. C. Velayudhan ${ }^{3}$, \\ Mirvaz Zulfiker ${ }^{3}$, Midhun M. ${ }^{3}$, Sanju Rajappan ${ }^{3}$, Sily Sreedharan ${ }^{4}$, Deeshma $^{4}$, \\ Remash K. ${ }^{3}$, Kanniyan Binub ${ }^{5 *}$
}

\author{
${ }^{1}$ Department of Pharmacology, Malabar Medical College and Research Center, Kerala, India \\ ${ }^{2}$ Internal Medicine, NMC Royal Clinic, Sharjah, United Arab Emirates \\ ${ }^{3}$ Department of General Medicine, Malabar Medical College and Research Center, Kerala, India \\ ${ }^{4}$ Department of Pathology, Malabar Medical College and Research Center, Kerala, India \\ ${ }^{5}$ Department of Community Medicine, Malabar Medical College and Research Center, Kerala, India
}

Received: 30 December 2021

Accepted: 14 January 2022

\author{
*Correspondence: \\ Dr. Kanniyan Binub, \\ E-mail: kanniyanbinub@gmail.com
}

Copyright: (C) the author(s), publisher and licensee Medip Academy. This is an open-access article distributed under the terms of the Creative Commons Attribution Non-Commercial License, which permits unrestricted non-commercial use, distribution, and reproduction in any medium, provided the original work is properly cited.

\begin{abstract}
Nanotechnology is the use of matter on an atomic, molecular, and supramolecular scale for various purposes. Nanotechnology field of application is very much diverse which includes surface science, organic chemistry, molecular biology, semiconductor physics, energy storage, engineering, microfabrication, and molecular engineering. Its medical application ranges from biological devices, nano-electronic biosensors, and to future biological machines. The main issue nowadays for nanomedicine involve understanding the issues related to toxicity and environmental impact of nanoscale materials. Lot more functionalities can be added to nanomaterials by interfacing them with biological structures. The size of nanomaterials is similar most biological molecules and so useful for both in vivo and in vitro biomedical research and applications. The integration of nanomaterials with biology had paved path to the development of diagnostic devices, contrast agents, analytical tools, physical therapy applications and drug delivery vehicles.
\end{abstract}

Keywords: Nanoparticle, Cancer, Chemotherapy

\section{INTRODUCTION}

In recent days, biomedical research has evolved and focused on the field of nanotechnology, which applies to various medical sectors such as diagnosis, monitoring, bioimaging and cure of the disease. Nanotechnology deals with the matter size range from 1 to $1000 \mathrm{~nm}^{1}$

The application of nanotechnology in the field of medicine, known as Nanomedicine is a novel area for diagnostic and therapeutic exploration.

The American physician Richard Feynman introduced this concept in 1959. Ensuing many scientists explored different ways for the development of nanostructures.
Subsequently growth of nanomedicine was tremendous which led to the development of liposomes, DNA-drug complexes, polymer-drug conjugates, antibody-drug conjugates, polymer nanocapsules, anti-arthritis gold nanoparticles and anti-microbial silver nanoparticles etc.

The advantage of Nanoparticles (NP) comprise its ultrasmall size, higher surface area, improved pharmacokinetic property, enabled target-specific drug delivery and increased stability. The limitations include the toxic reactions due to the nanoparticles themselves and the arduous steps for the formulation of stabilized nanoparticle complexes. These nanoparticles can be formed from both biodegradable polymers and non-biodegradable polymers. Fullerenes, carbon nanotubes, nanowires, quantum dots 
and dendrimers are examples of the different types of nanoparticles.

\section{NANOPARTICLES CHEMOTHERAPY}

IN

CANCER

The rapid growth in the field of nanotechnology towards the synthesis and development of nanomedicine agents hold a massive potential in the field of cancer chemotherapy. The studies showed that particles in the diameter size range of $10-100 \mathrm{~nm}$ are generally suitable for cancer chemotherapy as they have an enhanced permeability and retention effect.

The organic NPs (liposome-based, polymer based and dendrimers), inorganic NPs (carbon nanotubes, silica and magnetic-based and quantum dots) and hybrid NPs are available for cancer chemotherapy. Liposomes, a form of vesicles that consist of many or few or one phospholipid bilayers provide an effective drug delivery rostrum for different chemotherapeutic agents such as doxorubicin, paclitaxel etc. ${ }^{2}$

Paclitaxel liposomes have been proven their superiority in the antitumor activity and bioavailability over free paclitaxel. Liposomal doxorubicin showed reduced cardiotoxicity and comparable efficacy in the treatment of breast cancer when compared to free doxorubicin. Nowadays numerous liposomal based therapies are available for clinical use. Polylactic-co-glycolic acid (PLGA) a widely used polymer-based NP is formed by the copolymerization of lactic acid and glycolic acid. ${ }^{3}$ Better biocompatibility and degradation are the trump card features of polymer-based NPs. Dendrimers are characterized by a three-dimensional branched structure consist of a hydrophilic segment that enhances the stability and a hydrophobic segment for the smooth delivery of anticancer agents.

Even though poor biocompatibility and biodegradability are the major drawbacks of the Inorganic NPs they possess a higher surface area to volume ratio and they have easily modifiable surface chemistry. Among the inorganic NPs, the gold NPs (AuNPs) are mostly studied and involved in multimodal cancer therapies such as gene therapy, immunotherapy etc. Carbon nanotubes consist of tubular material and have been used to deliver drugs such as doxorubicin, paclitaxel and methotrexate. Exposure of carbon nanotubes to infrared beams may produce intense thermal energy which is useful for the ablation of cancer. ${ }^{4}$

Silica NPs are with a large internal pore volume which enables them to contain a larger amount of anti-cancer agents and they have a better pharmacokinetic and treatment efficacy. Also, the promotion of antigen crosspresentation, the polarization of lymphocytes and secretion of Interferon- $\gamma($ IFN- $\gamma$ ) makes silica NPs an ideal candidate for cancer immunotherapy. Magnetic NPs contains metals or metal oxides besides a coating with fatty acids or polymer for improved stability. They have shown high efficacy in cancer chemotherapy and gene therapy. ${ }^{5}$ The combination of organic and inorganic nanoparticles is the commonly practised hybrid NP design.

A hybrid NP carrier with better biological properties offers an enhanced drug treatment efficacy and reduced drug resistance. For example, a Liposome-silica hybrid (LSH) has been provided with a platform for the synergistic delivery of gemcitabine and paclitaxel for the treatment of pancreatic cancer in the mouse model of the disease. Carbon nanotubes and chitosan hybrid NPs enhanced the antitumor activity of methotrexate in lung cancer and reduced the toxicity in the normal cells.

The targeting designs using the NPs in cancer chemotherapy constitute active and passive designs. The targeting offers enhanced specificity, greater efficacy and lesser systemic toxicity. Passive targeting depends on many factors such as neovascularization, defective angiogenesis and poor lymphatic drainage, which grants an increase in the retention of NPs at the cancer cells.

This will contribute to a greater efficacy with the use of NPs. Unlike passive targeting, active targeting directly deals with the interaction between the ligands present on the NPs and receptors present on the tumour cells. The receptor-mediated endocytosis will favour the entry of NPs within the cell and aid in the release of chemotherapeutic agents. ${ }^{6}$ For example, transferrin receptors are overexpressed in most solid tumours so transferrin conjugated delivery of NPs with the chemotherapeutic agent may yield greater specificity, cellular uptake and greater efficacy.

Drug resistance is a major barrier in the field of cancer chemotherapy. The defective apoptotic machinery promotes the survival and growth of cancer therapy. Bcl-2 is an antiapoptotic protein highly expressed in most tumours. Researches proved theco-delivery of Bcl-2targeted siRNA and chemotherapeutics as an alternative to overcome the drug resistance. ${ }^{7}$

Overexpression of hypoxia-inducible factor $1 \alpha$ (HIF-1 $\alpha)$ produces multidrug resistance in many human cancers. Heat shock protein (HSP90) is an essential factor for the transcriptional activity of HIF-1 $\alpha$. Studies showed that administration of The HSP90 inhibitor in 17AAG-loaded NPs improved the bladder cancer treatment outcome.

The application of nanotechnology has led to a newer revolutionary phase in cancer treatment. When compared to the traditional drugs alone the NPS-based drug delivery provides better pharmacokinetics, biocompatibility, tumour targeting, and stability.

Moreover, they reduce drug toxicity in the normal cells and also help to overcome drug resistance. The exponential use of these NP based agents provides minimum collateral damage and better therapeutic targeting. 
Moreover, nanovaccines and nanoparticle-based immunotherapy are all on the way, to make a newer era in the field of cancer therapy.

\section{NANOPARTICLES IN NEURODEGENERATIVE DISORDERS}

In recent decades, the improvement of lifestyle and quality of life increased the average life expectancy of the population even in the developing countries, which indirectly contributed to the increased incidence of neurodegenerative disorders. Neuroinflammation, an inflammatory reaction of the central nervous system is the hallmark of most neurodegenerative diseases.

Neurodegeneration, an irreversible loss of neurons is the cause of so many disorders like Parkinson's disease (PD), Huntington's disease (HD), and Alzheimer's disease (AD) etc. The present treatment plan strategies available could improve the symptoms and slow the progression of the disease. The main challenge in the pharmacotherapy of neurogenerative disorders is the formation of therapeutic agents that can pass the Blood-brain barrier (BBB) effectively. Nanotechnology is a promising area that can be used for the management of neurogenerative disorders. The NPs can be used as a vehicle across the BBB and can be used to inhibit the neuroinflammatory reactions within the CNS. ${ }^{8}$

$\mathrm{AD}$ is the most devastating disorder especially in the elder population all around the world and is one of the major causes of dementia. The most common proposed molecular mechanism is the hyperactivation of Cyclindependent kinase-5 (CDK5) and tau hyperphosphorylation which collectively leads to the formation and accumulation of Neurofibrillary tangles (NFTs). The abnormal processing of Amyloid precursor protein (APP) and reduction in calcium signalling also contributes to the disease progress. Curcumin is a natural antioxidant that can reduce tau protein hyperphosphorylation and reduce plaque accumulation. Because of this curcumin is an attractive treatment option in AD. The curcumin loaded PLGA NPs easily crossed BBB and showed protective nature against beta-amyloid plaque accumulation. ${ }^{9}$

The preclinical studies conducted in the transgenic mice of the AD model showed a reduction in the formation of an amyloid-beta protein with Curcumin and Selenium nanospheres (Se-Cur poly D, L-lactic acid Co-Glycolic acid). Silver nanoparticle combined with aqueous extraction of Lampranthuscoccineus, Malephora lutea, F. aizoaceae administered in adult male Albino rats of Sprague-Dawley showed improved anticholinesterase and antioxidant property.

Niosomes are stable non-ionic surfactant-based vesicles in the NPs size range. Rivastigmine, an acetylcholine esterase inhibitor administered in the form of noisome showed greater BBB permeability with minimal side effects. ${ }^{10}$
PD is the second most common neurodegenerative disorder characterized by the degeneration of the dopaminergic neurons particularly in the area of substantia nigra and aggregation of $\alpha$-synuclein in the form of levy bodies. Conventional drug therapies such as L-Dopa has many limitations. Presently pharmacotherapy for PD with the aid of nanotechnology is in the evolving phase. Research using the Dopamine-PEGylated immunoliposomes (DA-PILs) showed 8 fold increase in the uptake when compared to the dopamine in the rat model of PD. ${ }^{11}$ Administration of Cerium oxide $\left(\mathrm{CeO}_{2}\right)$ NPs showed neuroprotective efficacy by exerting antioxidant activity in PD. A study conducted in a rotenone-induced animal model of $\mathrm{PD}$ with polymeric loaded NP loaded with ropinirole showed decreased neurodegeneration and astrogliosis. In another study using mice models of PD administration of a chitosan nanosuspension to co-deliver the PD drugs, pramipexole and hesperidin (an antioxidant) showed reduced degeneration. In the mouse model of PD intravenous administration of chitosan-coated levodopa, liposomes helped to reduce motor disturbances when compared to conventional levodopa tablets. Another research study showed that cerium NPs could protect the substantia nigra dopaminergic neurons against MPTP toxin, which can induce PD. ${ }^{12}$

HD is also a progressive neurodegenerative disorder because of the genetic abnormalities, metabolic and nutritional anomalies. The administration of Seleniumbased NP (Se-NP) in transgenic HD models of Caenorhabditis Elegans reduced neuronal death, attenuated oxidative stress, down-regulated histone deacetylase expression. The findings showed that Se-NP drug delivery is a promising candidate in the future. In another study using Curcumin (a strong antioxidant) based NP therapy showed reduction of apoptosis in an in vitro model of HD.

Amyotrophic lateral sclerosis (ALS) is also a neurodegenerative disorder characterized by the degeneration of upper motor and lower motor neurons. In a work by Antonello Spinelli and colleagues, Poly Ethylene Glycol coated AuNP administration in mice enhanced brain penetrance and improved stability of the neurons. So, the incorporation of conventional agents with NPs is a future rising target for the management of ALS. Multiple sclerosis (MS) is another demyelinating disease characterized by inflammatory demyelination within the CNS. The present therapies are focused to control the immune attack against the myelin sheath. Previous works of the literature showed that gold plated nanocrystals could fight MS progression and nerve damage.

Baclofen loaded nano lipid particles with the loaded drug were found effective for MS, whereas baclofen reduces spasticity. ${ }^{13}$ The works done by Gandomi et al.showed pegylated Solid lipid nanoparticles (SLN) nanoparticles loaded with methylprednisolone are effective for the management of MS. 


\section{CONCLUSION}

The NPs has a promising future in the development of neurodegenerative disorders and cancer chemotherapy. The advantage is that peculiar NP can be designed and developed based on the underlying pathology of the disease. So, agents with BBB penetration, improved pharmacokinetic properties, lesser toxicity and tolerance can be developed. Moreover, multiple agents such as multiple molecules, genes, therapeutics agents can be administered simultaneously with the aid of NP assistance. In recent decades nano, particle-based liposomes and polymers were approved for clinical use and so many newer agents are in the pipeline. In future, the rapid growth in nanotechnology and science will bring more development of polymers with an improved therapeutic approach in the field of cancer chemotherapy and neurodegenerative diseases.

Funding: No funding sources

Conflict of interest: None declared

Ethical approval: Not required

\section{REFERENCES}

1. Murthy SK. Nanoparticles in modern medicine: state of the art and future challenges. Int $\mathrm{J}$ Nanomedicine. 2007;2(2):129-41.

2. Zylberberg C, Matosevic S. Pharmaceutical liposomal drug delivery: a review of new delivery systems and a look at the regulatory landscape. Drug Deliv. 2016;23(9):3319-29.

3. Acharya S, Sahoo SK. PLGA nanoparticles containing various anticancer agents and tumour delivery by EPR effect. Adv Drug Deliv Rev. 2011;63(3):170-83.

4. Luo E, Song G, Li Y, Shi P, Hu J, Lin Y. The toxicity and pharmacokinetics of carbon nanotubes as an effective drug carrier. Curr Drug Metab. 2013;14(8):879-90.

5. Mandriota G, Corato R, Benedetti M, Castro F, Fanizzi FP, Rinaldi R. Design and Application of Cisplatin-Loaded Magnetic Nanoparticle Clusters for
Smart Chemotherapy. ACS Appl Mater Interfaces. 2019;11(2):1864-75.

6. Farokhzad OC, Langer R. Impact of nanotechnology on drug delivery. ACS Nano. 2009;3(1):16-20.

7. Choi KY, Correa S, Min J, Li J, Roy S, Laccetti KH, et al. Binary Targeting of siRNA to Hematologic Cancer Cells In Vivo using Layer-by-Layer Nanoparticles. Adv Funct Mater. 2019;29(20):1900018.

8. Zhu FD, Hu YJ, Yu L, Zhou XG, Wu JM, Tang Y, et al. Nanoparticles: A Hope for the Treatment of Inflammation in CNS. Front Pharmacol. 2021;12:683935.

9. Springer. Curcumin-loaded polymeric nanoparticles for neuroprotection in neonatal rats with hypoxicischemic encephalopathy, 2021. Available at: https://link.springer.com/article/10.1007\%2Fs 12274 -018-2104-y. Accessed on 18 December 2021.

10. Estabragh MAR, Hamidifar Z, Pardakhty A. Formulation of Rivastigmine Niosomes for Alzheimer Disease. Int Pharmacy Acta. 2018;1(1):101-4.

11. Kang YS, Jung HJ, Oh JS, Song DY. Use of PEGylated Immunoliposomes to Deliver Dopamine Across the Blood-Brain Barrier in a Rat Model of Parkinson's Disease. CNS Neurosci Ther. 2016;22(10):817-23.

12. The Michael $\mathbf{J}$ Foundation. Cerium Oxide Nanoparticles in Treatment of Parkinson's Disease, 2021. Available at: https://www.michaeljfox. org/grant/ceriumoxidenanoparticles-treatmentparkinsons-disease. Accessed on 18 December 2021.

13. Chountoulesi M, Demetzos C. Promising Nanotechnology Approaches in Treatment of Autoimmune Diseases of Central Nervous System. Brain Sci. 2020;10(6):338.

Cite this article as: Chandran $\mathrm{A}$, Raghavan V, Chalil B, Kamalasanan, Velayudhan CC, Zulfiker M, Midhun M, et al. Nanoparticles: tech trends in healthcare. Int J Res Med Sci 2022;10:576-9. 